\title{
UCRL-JRNL-204072
}

LAWRENCE LIVERMORE NATIONAL LABORATORY

\section{Translationally Invariant Density}

P. Navratil

May 11, 2004

Physical Review C 


\section{Disclaimer}

This document was prepared as an account of work sponsored by an agency of the United States Government. Neither the United States Government nor the University of California nor any of their employees, makes any warranty, express or implied, or assumes any legal liability or responsibility for the accuracy, completeness, or usefulness of any information, apparatus, product, or process disclosed, or represents that its use would not infringe privately owned rights. Reference herein to any specific commercial product, process, or service by trade name, trademark, manufacturer, or otherwise, does not necessarily constitute or imply its endorsement, recommendation, or favoring by the United States Government or the University of California. The views and opinions of authors expressed herein do not necessarily state or reflect those of the United States Government or the University of California, and shall not be used for advertising or product endorsement purposes. 


\title{
Translationally invariant density
}

\author{
Petr Navrátil \\ Lawrence Livermore National Laboratory, L-414, P.O. Box 808, Livermore, CA 94551
}

\begin{abstract}
Translationally invariant nuclear density is derived from the shell model one-body densities by removing their spurious $0 \hbar \Omega$ center-of-mass motion component. This paves the way to utilize the $a b$ initio no-core shell model (NCSM) nuclear structure in folding approaches to optical potentials. As an illustration, the ${ }^{6} \mathrm{He}$ diagonal and transitional densities are calculated from the NCSM wave functions obtained using the CD-Bonn nucleon-nucleon potential in the $10 \hbar \Omega$ basis space. A particularly significant impact of the exact removal of the spurious center-of-mass motion is found for the spin-orbit part of the optical potential proportional to the derivative of the nuclear density.
\end{abstract}

PACS numbers: 21.60.Cs, 24.50.+g, 21.30.-x, 21.30.Fe

\section{INTRODUCTION}

There has been a significant progress in the ab initio approaches to the structure of light nuclei. Starting from the realistic two- and three-nucleon interactions the methods like the Green's Function Monte Carlo (GFMC) [1] or the $a b$ initio no-core shell model (NCSM) [2] can predict the low-lying levels in $p$-shell nuclei.

The principal foundation of the $a b$ initio NCSM approach is the use of effective interactions appropriate for the large but finite basis spaces employed in the calculations. These effective interactions are derived from the underlying realistic inter-nucleon potentials through a unitary transformation in a way that guarantees convergence to the exact solution as the basis size increases. For the basis, one uses antisymmetrized $A$-nucleon harmonic-oscillator (HO) states that span the complete $N_{\max } \hbar \Omega$ space. A disadvantage of the $\mathrm{HO}$ basis is its unphysical asymptotic behavior. On the other hand, the nuclear system is translationally invariant and, in particular in the case of light nuclei, it is important to preserve this symmetry. The HO basis is the only basis that allows to switch from Jacobi coordinates to single particle Cartesian coordinates without violating the translational invariance. Consequently, one may choose the coordinates according to whatever is more efficient for the problem at hand. In practice, it turns out that the $A=3$ system is the easiest solved in the Jacobi basis, the $A=4$ system can be solved either way with the same efficiency when only two-body interaction is utilized, but the Jacobi basis is more efficient when the three-body interaction is included. For systems with $A>4$, it is by far more advantageous to use the Cartesian coordinates and the Slater determinant (SD) basis and employ the powerful shell model codes like Antoine [3] that rely on the second quantization techniques.

While the NCSM eigenenergies are independent on the choice of coordinates, the eigenfunctions obtained in the Cartesian coordinate SD basis include a $0 \hbar \Omega$ spurious center of mass component. The ways how to remove these components and obtain physical matrix elements of different operators were investigated in the past [4-10]. Typically, in earlier investigations the basis space was limited to a single major HO shell. In the NCSM, the basis space spans several major shells. Unlike in some phenomenological shell-model studies that used a multi-major shell basis, the center-of-mass motion is completely separated from the internal motion due to the translational invariance of the interactions and the choice of the complete $N_{\max } \hbar \Omega \mathrm{HO}$ basis as already discussed. In general, it is necessary to re-visit and adapt the techniques of the spurious center-of-mass (CM) motion removal to make them applicable for the NCSM. This paper in particular focuses on the construction of the translationally invariant density starting from the Cartesian coordinate SD wave functions. This case is much less trivial than, e.g. removal of spurious components from spectroscopic amplitudes.

The motivation for this work is the desire to apply the ab initio NCSM nuclear structure to describe nuclear reactions on light nuclei. In general, it is a challenging task to extend the $a b$ initio methods to describe nuclear reactions. Concerning direct reactions, in particular the nucleon-nucleus elastic and inelastic scattering, a first and straightforward answer for the NCSM is the application of semi-microscopic approaches, e.g. the Jeukenne-LejeuneMahaux (JLM) [11], to construct optical potentials from the nuclear densities obtained in the NCSM. Eventually, these optical potentials are used in coupled channel calculations by employing the standard codes, e.g. Fresco [12]. To fully utilize the NCSM nuclear structure for this purpose, the spurious center-of-mass contribution must be removed from the density. In Sec. II, the translational invariant density is derived from both the Jacobi-coordinate HO wave functions as well as from the Cartesian-coordinate wave functions. In Sec. III, numerical tests for $A=3,4$ and 5 systems are described and an application to ${ }^{6} \mathrm{He}$ is presented. A spin-orbit part of the $p+{ }^{6} \mathrm{He}$ optical potential is constructed to demonstrate the importance of the spurious center-of-mass removal. Conclusions are given in Sec. IV. 


\section{DERIVATION OF THE TRANSLATIONALLY INVARIANT DENSITY}

\section{A. Coordinate and HO wave function transformations}

We follow the notation of Ref. [13]. We consider nucleons with the mass $m$ neglecting the difference between the proton and the neutron mass. For the purpose of the present paper we use the following set of Jacobi coordinates:

$$
\begin{aligned}
& \vec{\xi}_{0}=\sqrt{\frac{1}{A}}\left[\vec{r}_{1}+\vec{r}_{2}+\ldots+\vec{r}_{A}\right] \\
& \vec{\xi}_{1}=\sqrt{\frac{1}{2}}\left[\vec{r}_{1}-\vec{r}_{2}\right] \\
& \vec{\xi}_{2}=\sqrt{\frac{2}{3}}\left[\frac{1}{2}\left(\vec{r}_{1}+\vec{r}_{2}\right)-\vec{r}_{3}\right] \text {, } \\
& \vec{\xi}_{A-2}=\sqrt{\frac{A-2}{A-1}}\left[\frac{1}{A-2}\left(\vec{r}_{1}+\vec{r}_{2}+\ldots+\vec{r}_{A-2}\right)-\vec{r}_{A-1}\right] \text {, } \\
& \vec{\xi}_{A-1}=\sqrt{\frac{A-1}{A}}\left[\frac{1}{A-1}\left(\vec{r}_{1}+\vec{r}_{2}+\ldots+\vec{r}_{A-1}\right)-\vec{r}_{A}\right] \text {. }
\end{aligned}
$$

Here, $\vec{\xi}_{0}$ is proportional to the center of mass of the $A$-nucleon system: $\vec{R}=\sqrt{\frac{1}{A}} \vec{\xi}_{0}$. On the other hand, $\vec{\xi}_{\rho}$ is proportional to the relative position of the $\rho+1$-st nucleon and the center of mass of the $\rho$ nucleons. Let us rewrite the last and the first equation from (1) as

$$
\begin{aligned}
\vec{\xi}_{A-1} & =\sqrt{\frac{1}{A}} \vec{R}_{\mathrm{CM}}^{A-1}-\sqrt{\frac{A-1}{A}} \vec{r}_{A}, \\
\vec{\xi}_{0} & =\sqrt{\frac{A-1}{A}} \vec{R}_{\mathrm{CM}}^{A-1}+\sqrt{\frac{1}{A}} \vec{r}_{A},
\end{aligned}
$$

where $\vec{R}_{\mathrm{CM}}^{A-1}=\sqrt{\frac{1}{A-1}}\left[\vec{r}_{1}+\vec{r}_{2}+\ldots+\vec{r}_{A-1}\right]$. Following, e.g. Ref. [14], the HO wave functions depending on the coordinates (2) transform as

$$
\begin{aligned}
& \sum_{M_{1} m_{1}}\left(L_{1} M_{1} l_{1} m_{1} \mid Q q\right) \varphi_{N_{1} L_{1} M_{1}}\left(\vec{R}_{\mathrm{CM}}^{A-1}\right) \varphi_{n_{1} l_{1} m_{1}}\left(\vec{r}_{A}\right)= \\
& \sum_{n l m N L M}\left\langle n l N L Q \mid N_{1} L_{1} n_{1} l_{1} Q\right\rangle \frac{1}{A-1}(l m L M \mid Q q) \varphi_{n l m}\left(\vec{\xi}_{A-1}\right) \varphi_{N L M}\left(\vec{\xi}_{0}\right)
\end{aligned}
$$

where $\left\langle n l N L Q \mid N_{1} L_{1} n_{1} l_{1} Q\right\rangle_{\frac{1}{A-1}}$ is the general HO bracket for two particles with mass ratio $\frac{1}{A-1}$.

\section{B. Nuclear density}

Nuclear density operator is defined as [15]

$$
\rho_{o p}(\vec{r})=\sum_{i=1}^{A} \delta\left(\vec{r}-\vec{r}_{i}\right)=\sum_{i=1}^{A} \frac{\delta\left(r-r_{i}\right)}{r r_{i}} \sum_{m} Y_{l m}\left(\hat{r}_{i}\right) Y_{l m}^{*}(\hat{r}) .
$$

Its matrix element between an initial and a final state that were obtained in Cartesian coordinate single-particle SD basis can be written in the form

$$
\begin{aligned}
\mathrm{SD}\left\langle A \lambda_{f} J_{f} M_{f}\left|\rho_{o p}(\vec{r})\right| A \lambda_{i} J_{i} M_{i}\right\rangle_{\mathrm{SD}} & =\frac{1}{\hat{J}_{f}} \sum\left(J_{i} M_{i} K k \mid J_{f} M_{f}\right) Y_{K k}^{*}(\hat{r}) R_{n_{1} l_{1}}(r) R_{n_{2} l_{2}}(r)\left\langle l_{1} \frac{1}{2} j_{1}\left\|Y_{K}\right\| l_{2} \frac{1}{2} j_{2}\right\rangle \\
& \times \frac{-1}{\hat{K}} \mathrm{SD}\left\langle A \lambda_{f} J_{f}\left\|\left(a_{n_{1} l_{1} j_{1}}^{\dagger} \tilde{a}_{n_{2} l_{2} j_{2}}\right)^{(K)}\right\| A \lambda_{i} J_{i}\right\rangle_{\mathrm{SD}} .
\end{aligned}
$$

Here, $|A \lambda J M\rangle_{\mathrm{SD}}$ is an $A$-nucleon eigenstate with the angular momentum $J$ and its third component $M, \hat{K}=\sqrt{2 K+1}$ and $\tilde{a}_{j m}=(-1)^{j-m} a_{j,-m}$. The $\lambda$ stands for remaining quantum numbers. The subscript SD refers to the fact 
that this state was obtained in the Slater determinant basis, i.e. by using a shell model code, and, consequently, contains the spurious $\mathrm{CM}$ component. The $R_{n l}(r)$ in Eq. (5) is the radial HO wave function and the term $\frac{-1}{\hat{K}} \mathrm{SD}\left\langle A \lambda_{f} J_{f}\left\|\left(a_{n_{1} l_{1} j_{1}}^{\dagger} \tilde{a}_{n_{2} l_{2} j_{2}}\right)^{(K)}\right\| A \lambda_{i} J_{i}\right\rangle_{\mathrm{SD}}$ represents the standard one-body density matrix elements (OBDME) computed in shell model codes. The coordinate $\vec{r}$ in (5) is measured from the center of the HO potential well. Clearly, the density given in (5) contains a contribution from the spurious CM motion.

The physical density should depend on the coordinate measured from the CM of the nucleus, $\vec{r}-\vec{R}$. The corresponding matrix element is obtained by employing the eigenstates depending on the Jacobi coordinates. By modifying the last relation in (4) we get

$$
\left\langle A \lambda_{f} J_{f} M_{f}\left|\rho_{o p}(\vec{r}-\vec{R})\right| A \lambda_{i} J_{i} M_{i}\right\rangle=A\left(\frac{A}{A-1}\right)^{3 / 2}\left\langle A \lambda_{f} J_{f} M_{f}\left|\delta\left(\vec{\xi}-\vec{\xi}_{A-1}\right)\right| A \lambda_{i} J_{i} M_{i}\right\rangle,
$$

where $\vec{\xi}=-\sqrt{\frac{A}{A-1}}(\vec{r}-\vec{R})$ and $\vec{\xi}_{A-1}$ given by Eq. (1e) is re-expressed as $\vec{\xi}_{A-1}=-\sqrt{\frac{A}{A-1}}\left(\vec{r}_{A}-\vec{R}\right)$. We used the antisymmetry of the eigenstates and the properties of the Dirac delta function. The relationship between the Jacobi coordinate and the SD eigenstates is

$$
\left\langle\vec{r}_{1} \ldots \vec{r}_{A} \sigma_{1} \ldots \sigma_{A} \tau_{1} \ldots \tau_{A} \mid A \lambda J M\right\rangle_{\mathrm{SD}}=\left\langle\vec{\xi}_{1} \ldots \vec{\xi}_{A-1} \sigma_{1} \ldots \sigma_{A} \tau_{1} \ldots \tau_{A} \mid A \lambda J M\right\rangle \varphi_{000}\left(\vec{\xi}_{0}\right),
$$

with the $\sigma$ and $\tau$ the spin and isospin coordinates, respectively.

Similarly as in (5), the physical density (6) can be related to "one-body" density matrix elements derived from the Jacobi coordinate eigenstates (discussed, e.g. in the Appendix B of Ref. [13]). In particular, we obtain

$$
\begin{aligned}
\left\langle A \lambda_{f} J_{f} M_{f}\left|\rho_{o p}(\vec{r}-\vec{R})\right| A \lambda_{i} J_{i} M_{i}\right\rangle & =A\left(\frac{A}{A-1}\right)^{3 / 2} \hat{J}_{i} \sum\left(J_{i} M_{i} K k \mid J_{f} M_{f}\right) Y_{K k}^{*}(\vec{r}-\vec{R}) \\
& \times R_{n l}\left(\sqrt{\frac{A}{A-1}}|\vec{r}-\vec{R}|\right) R_{n^{\prime} l^{\prime}}\left(\sqrt{\frac{A}{A-1}}|\vec{r}-\vec{R}|\right) \\
& \times\left\langle l \frac{1}{2} j \| Y_{K}|| l^{\prime} \frac{1}{2} j^{\prime}\right\rangle(-1)^{J_{\mathrm{A}-1}+J_{f}+j^{\prime}}\left\{\begin{array}{ccc}
J_{\mathrm{A}-1} & j^{\prime} & J_{i} \\
K & J_{f} & j
\end{array}\right\} \\
& \times\left\langle A \lambda_{f} J_{f} \mid\left(N_{\mathrm{A}-1} i_{\mathrm{A}-1} J_{\mathrm{A}-1} ; n l j\right) J_{f}\right\rangle\left\langle\left(N_{\mathrm{A}-1} i_{\mathrm{A}-1} J_{\mathrm{A}-1} ; n^{\prime} l^{\prime} j^{\prime}\right) J_{i} \mid A \lambda_{i} J_{i}\right\rangle .
\end{aligned}
$$

Here the eigenstates are expanded in a basis with lower degree of antisymmetry using the coefficients of fractional parentage [13]

$$
\left\langle\left(N_{\mathrm{A}-1} i_{\mathrm{A}-1} J_{\mathrm{A}-1} ; n l j\right) J \mid A \lambda J\right\rangle=\sum\left\langle N_{\mathrm{A}-1} i_{\mathrm{A}-1} J_{\mathrm{A}-1} ; n l j|| N i J\right\rangle\langle N i J \mid A \lambda J\rangle,
$$

with $N=N_{\mathrm{A}-1}+2 n+l$ the total number of $\mathrm{HO}$ excitations for the $A$ nucleons and $i, i_{\mathrm{A}-1}$ the additional quantum numbers that characterize the $A$ - and $A$-1-nucleon antisymmetrized basis states, respectively.

\section{Physical density in terms of the SD OBDME}

It turns out that obtaining the eigenstates (9) becomes increasingly difficult with the number of nucleons $A$ mostly due to the complicated antisymmetrization. As stated in the Introduction, for $A>4$ it is by far more efficient to use the SD basis. Consequently, it is desirable to relate the matrix element (6) to the SD OBDME $\frac{-1}{\hat{K}} \mathrm{SD}\left\langle A \lambda_{f} J_{f}\left\|\left(a_{n_{1} l_{1} j_{1}}^{\dagger} \tilde{a}_{n_{2} l_{2} j_{2}}\right)^{(K)}\right\| A \lambda_{i} J_{i}\right\rangle_{\mathrm{SD}}$.

Let us note that an intermediate result that eventually leads to the right-hand side of Eq. (8) reads:

$$
\begin{aligned}
& \left\langle A \lambda_{f} J_{f} M_{f}\left|\rho_{o p}(\vec{r}-\vec{R})\right| A \lambda_{i} J_{i} M_{i}\right\rangle=A\left(\frac{A}{A-1}\right)^{3 / 2} \sum R_{n l}(\xi) R_{n^{\prime} l^{\prime}}(\xi) \frac{\hat{l} \hat{l}^{\prime}}{\sqrt{4 \pi} \hat{K}^{\prime}}\left(l 0 l^{\prime} 0 \mid K 0\right) Y_{K k}^{*}(\hat{\xi})\left(l m l^{\prime} m^{\prime} \mid K k\right) \\
& \int d \vec{\xi}_{1} \ldots d \vec{\xi}_{A-2} d \vec{\xi}_{A-1} d \vec{\xi}_{A-1}^{\prime}\left\langle A \lambda_{f} J_{j} M_{f} \mid \vec{\xi}_{1} \ldots \vec{\xi}_{A-1}\right\rangle \varphi_{n l m}\left(\vec{\xi}_{A-1}\right) \varphi_{n^{\prime} l^{\prime} m^{\prime}}\left(\vec{\xi}_{A-1}^{\prime}\right)\left\langle\vec{\xi}_{1} \ldots \vec{\xi}_{A-2} \vec{\xi}_{A-1}^{\prime} \mid A \lambda_{i} J_{i} M_{i}\right\rangle,
\end{aligned}
$$

where for the simplicity we suppress from now on spin and isospin coordinates. In deriving Eq. (10) we used the Dirac delta function properties and the relation $\delta\left(\vec{\xi}-\vec{\xi}_{A-1}\right)=\sum_{n l m} \varphi_{n l m}\left(\vec{\xi}_{A-1}\right) \varphi_{n l m}^{*}(\vec{\xi})$.

We now investigate an analogous integral to that appearing on the right-hand side of Eq. (10) for the Cartesian coordinate wave functions and, as the first result, we relate it to the OBDME:

$$
\begin{aligned}
& \sum_{\sigma_{i} \tau_{i} m_{1} m_{2}}\left(l_{1} m_{1} l_{2} m_{2} \mid K k\right) \int d \vec{r}_{1} \ldots d \vec{r}_{A-1} d \vec{r}_{A} d \vec{r}_{A \mathrm{SD}}^{\prime}\left\langle A \lambda_{f} J_{f} M_{f} \mid \vec{r}_{1} \ldots \vec{r}_{A}\right\rangle \varphi_{n_{1} l_{1} m_{1}}\left(\vec{r}_{A}\right) \varphi_{n_{2} l_{2} m_{2}}\left(\vec{r}_{A}^{\prime}\right) \\
& \times\left\langle\vec{r}_{1} \ldots \vec{r}_{A-1} \vec{r}_{A}^{\prime} \mid A \lambda_{i} J_{i} M_{i}\right\rangle_{\mathrm{SD}}= \\
& \frac{1}{A} \sum_{j_{1} j_{2} \mathrm{SD}}\left\langle A \lambda_{f} J_{f} M_{f}\left|\left(a_{n_{1} l_{1} j_{1}}^{\dagger} \tilde{a}_{n_{2} l_{2} j_{2}}\right)_{k}^{(K)}\right| A \lambda_{i} J_{i} M_{i}\right\rangle_{\mathrm{SD}}(-1)^{l_{1}+l_{2}+K+j_{2}-\frac{1}{2}} \hat{j}_{1} \hat{j}_{2}\left\{\begin{array}{ccc}
j_{1} & j_{2} & K \\
l_{2} & l_{1} & \frac{1}{2}
\end{array}\right\} .
\end{aligned}
$$


Next, we re-write the left-hand-side of Eq. (11) and perform a change of variables to the Jacobi coordinates,

$$
\begin{aligned}
& \sum_{\sigma_{i} \tau_{i} m_{1} m_{2}}\left(l_{1} m_{1} l_{2} m_{2} \mid K k\right) \int d \vec{r}_{1} \ldots d \vec{r}_{A-1} d \vec{r}_{A} d \vec{r}_{1}^{\prime} \ldots d \vec{r}_{A-1}^{\prime} d \vec{r}_{A \mathrm{SD}}^{\prime}\left\langle A \lambda_{f} J_{f} M_{f} \mid \vec{r}_{1} \ldots \vec{r}_{A}\right\rangle \varphi_{n_{1} l_{1} m_{1}}\left(\vec{r}_{A}\right) \varphi_{n_{2} l_{2} m_{2}}\left(\vec{r}_{A}^{\prime}\right) \\
& \times \delta\left(\vec{r}_{1}-\vec{r}_{1}^{\prime}\right) \ldots \delta\left(\vec{r}_{A-1}-\vec{r}_{A-1}^{\prime}\right)\left\langle\vec{r}_{1}^{\prime} \ldots \vec{r}_{A-1}^{\prime} \vec{r}_{A}^{\prime} \mid A \lambda_{i} J_{i} M_{i}\right\rangle_{\mathrm{SD}}= \\
& \sum_{\sigma_{i} \tau_{i} m_{1} m_{2}}\left(l_{1} m_{1} l_{2} m_{2} \mid K k\right) \int d \vec{\xi}_{1} \ldots d \vec{\xi}_{A-2} d \vec{R}_{\mathrm{CM}}^{A-1} d \vec{r}_{A} d \vec{\xi}_{1}^{\prime} \ldots d \vec{\xi}_{A-2}^{\prime} d \vec{R}_{\mathrm{CM}}^{\prime A-1} d \vec{r}_{A}^{\prime}\left\langle A \lambda_{f} J_{f} M_{f} \mid \vec{\xi}_{1} \ldots \vec{\xi}_{A-1}\right\rangle \varphi_{000}^{*}\left(\vec{\xi}_{0}\right) \\
& \times \varphi_{n_{1} l_{1} m_{1}}\left(\vec{r}_{A}\right) \varphi_{n_{2} l_{2} m_{2}}\left(\vec{r}_{A}^{\prime}\right) \delta\left(\vec{\xi}_{1}-\vec{\xi}_{1}^{\prime}\right) \ldots \delta\left(\vec{\xi}_{A-2}-\vec{\xi}_{A-2}^{\prime}\right) \delta\left(\vec{R}_{\mathrm{CM}}^{A-1}-\vec{R}_{\mathrm{CM}}^{\prime A-1}\right) \\
& \times \varphi_{000}\left(\vec{\xi}_{0}^{\prime}\right)\left\langle\vec{\xi}_{1}^{\prime} \ldots \vec{\xi}_{A-1}^{\prime} \mid A \lambda_{i} J_{i} M_{i}\right\rangle= \\
& \sum_{\sigma_{i} \tau_{i} m_{1} m_{2}}\left(l_{1} m_{1} l_{2} m_{2} \mid K k\right) \int d \vec{\xi}_{1} \ldots d \vec{\xi}_{A-2} d \vec{R}_{\mathrm{CM}}^{A-1} d \vec{r}_{A} d \vec{R}_{\mathrm{CM}}^{\prime A-1} d \vec{r}_{A}^{\prime}\left\langle A \lambda_{f} J_{f} M_{f} \mid \vec{\xi}_{1} \ldots \vec{\xi}_{A-1}\right\rangle \varphi_{000}^{*}\left(\vec{\xi}_{0}\right) \\
& \times \varphi_{n_{1} l_{1} m_{1}}\left(\vec{r}_{A}\right) \varphi_{N_{1} L_{1} M_{1}}\left(\vec{R}_{\mathrm{CM}}^{A-1}\right)(-1)^{m_{2}} \varphi_{n_{2} l_{2}-m_{2}}^{*}\left(\vec{r}_{A}^{\prime}\right) \varphi_{N_{1} L_{1} M_{1}}^{*}\left(\vec{R}_{\mathrm{CM}}^{\prime A-1}\right) \\
& \times \varphi_{000}\left(\vec{\xi}_{0}^{\prime}\right)\left\langle\vec{\xi}_{1} \ldots \vec{\xi}_{A-2} \vec{\xi}_{A-1}^{\prime} \mid A \lambda_{i} J_{i} M_{i}\right\rangle= \\
& \quad \sum_{\sigma_{i} \tau_{i} n l m n^{\prime} l^{\prime} m^{\prime} N_{1} L_{1}}(-1)^{l+l^{\prime}+K+L_{1}}\left\{\begin{array}{ll}
l_{1} L_{1} & l \\
l^{\prime} & K \\
l_{2}
\end{array}\right\} \hat{l} \hat{l}^{\prime}\left\langle n l 00 l \mid N_{1} L_{1} n_{1} l_{1} l\right\rangle \frac{1}{A-1}\left\langle n^{\prime} l^{\prime} 00 l^{\prime} \mid N_{1} L_{1} n_{2} l_{2} l^{\prime}\right\rangle \frac{1}{A-1} \\
& \times\left(l m l^{\prime} m^{\prime} \mid K k\right) \int d \vec{\xi}_{1} \ldots d \vec{\xi}_{A-2} d \vec{\xi}_{A-1} d \vec{\xi}_{A-1}^{\prime}\left\langle A \lambda_{f} J_{f} M_{f} \mid \vec{\xi}_{1} \ldots \vec{\xi}_{A-1}\right\rangle \varphi_{n l m}\left(\vec{\xi}_{A-1}\right) \varphi_{n^{\prime} l^{\prime} m^{\prime}}\left(\vec{\xi}_{A-1}^{\prime}\right) \\
& \times\left\langle\vec{\xi}_{1} \ldots \vec{\xi}_{A-2} \vec{\xi}_{A-1}^{\prime} \mid A \lambda_{i} J_{i} M_{i}\right\rangle .
\end{aligned}
$$

In the above derivation, we used the relations $(1,2,3)$ and $(7)$ together with $\delta\left(\vec{R}_{\mathrm{CM}}^{A-1}-\vec{R}_{\mathrm{CM}}^{\prime A-1}\right)=$ $\sum_{N_{1} L_{1} M_{1}} \varphi_{N_{1} L_{1} M_{1}}\left(\vec{R}_{\mathrm{CM}}^{A-1}\right) \varphi_{N_{1} L_{1} M_{1}}^{*}\left(\vec{R}_{\mathrm{CM}}^{\prime A-1}\right)$. The last term in Eq. (12) contains the integral appearing on the righthand-side of Eq. (10). We can now relate this integral to the OBDME, i.e. the right-hand-side of Eq. (11). In order to do that we define a matrix

$$
\left(M^{K}\right)_{n_{1} l_{1} n_{2} l_{2}, n l n^{\prime} l^{\prime}}=\sum_{N_{1} L_{1}}(-1)^{l+l^{\prime}+K+L_{1}}\left\{\begin{array}{ccc}
l_{1} & L_{1} & l \\
l^{\prime} & K & l_{2}
\end{array}\right\} \hat{l}^{\prime}\left\langle n l 00 l \mid N_{1} L_{1} n_{1} l_{1} l\right\rangle_{\frac{1}{A-1}}\left\langle n^{\prime} l^{\prime} 00 l^{\prime} \mid N_{1} L_{1} n_{2} l_{2} l^{\prime}\right\rangle_{\frac{1}{A-1}} .
$$

As we are interested only in the case, when $(-)^{l+l^{\prime}+K}=(-1)^{l_{1}+l_{2}+K}=1$, the above definition assumes this restriction.

Then, by inverting the matrix $M^{K}$ we obtain

$$
\begin{aligned}
& \sum_{\sigma_{i} \tau_{i} m m^{\prime}}\left(l m l^{\prime} m^{\prime} \mid K k\right) \int d \vec{\xi}_{1} \ldots d \vec{\xi}_{A-2} d \vec{\xi}_{A-1} d \vec{\xi}_{A-1}^{\prime}\left\langle A \lambda_{f} J_{j} M_{f} \mid \vec{\xi}_{1} \ldots \vec{\xi}_{A-1}\right\rangle \varphi_{n l m}\left(\vec{\xi}_{A-1}\right) \varphi_{n^{\prime} l^{\prime} m^{\prime}}\left(\vec{\xi}_{A-1}^{\prime}\right) \\
& \times\left\langle\vec{\xi}_{1} \ldots \vec{\xi}_{A-2} \vec{\xi}_{A-1}^{\prime} \mid A \lambda_{i} J_{i} M_{i}\right\rangle=\frac{1}{A} \sum_{n_{1} l_{1} j_{1} n_{2} l_{2} j_{2}}\left(M^{K}\right)_{n l n^{\prime} l^{\prime}, n_{1} l_{1} n_{2} l_{2}}^{-1} \\
& \times{ }_{\mathrm{SD}}\left\langle A \lambda_{f} J_{f} M_{f}\left|\left(a_{n_{1} l_{1} j_{1}}^{\dagger} \tilde{a}_{n_{2} l_{2} j_{2}}\right)_{k}^{(K)}\right| A \lambda_{i} J_{i} M_{i}\right\rangle_{\mathrm{SD}}(-1)^{l_{1}+l_{2}+K+j_{2}-\frac{1}{2}} \hat{j}_{1} \hat{j}_{2}\left\{\begin{array}{ccc}
j_{1} & j_{2} & K \\
l_{2} & l_{1} & \frac{1}{2}
\end{array}\right\} .
\end{aligned}
$$

Eventually, with the help of

$$
\left\langle l_{1} \frac{1}{2} j_{1}|| Y_{K}|| l_{2} \frac{1}{2} j_{2}\right\rangle=\frac{1}{\sqrt{4 \pi}} \hat{j}_{1} \hat{j}_{2} \hat{l}_{1} \hat{l}_{2}(-1)^{j_{2}+\frac{1}{2}}\left(l_{1} 0 l_{2} 0 \mid K 0\right)\left\{\begin{array}{ccc}
j_{1} & j_{2} & K \\
l_{2} & l_{1} & \frac{1}{2}
\end{array}\right\}
$$

for the spherical harmonics matrix element, we arrive at the main result of this paper

$$
\begin{aligned}
\left\langle A \lambda_{f} J_{f} M_{f}\left|\rho_{o p}(\vec{r}-\vec{R})\right| A \lambda_{i} J_{i} M_{i}\right\rangle & =\left(\frac{A}{A-1}\right)^{3 / 2} \frac{1}{\hat{J}_{f}} \sum\left(J_{i} M_{i} K k \mid J_{f} M_{f}\right) Y_{K k}^{*}(\vec{r}-\vec{R}) \\
& \times R_{n l}\left(\sqrt{\frac{A}{A-1}}|\vec{r}-\vec{R}|\right) R_{n^{\prime} l^{\prime}}\left(\sqrt{\frac{A}{A-1}}|\vec{r}-\vec{R}|\right) \\
& \times(-1)^{K} \frac{\hat{l} \hat{l}^{\prime}\left(l 0 l^{\prime} 0 \mid K 0\right)}{\hat{l}_{1} \hat{l}_{2}\left(l_{1} 0 l_{2} 0 \mid K 0\right)}\left(M^{K}\right)_{n l n^{\prime} l^{\prime}, n_{1} l_{1} n_{2} l_{2}}^{-1}\left\langle l_{1} \frac{1}{2} j_{1} \| Y_{K}|| l_{2} \frac{1}{2} j_{2}\right\rangle \\
& \times \frac{-1}{\hat{K}} \mathrm{SD}\left\langle A \lambda_{f} J_{f}\left\|\left(a_{n_{1} l_{1} j_{1}}^{\dagger} \tilde{a}_{n_{2} l_{2} j_{2}}\right)^{(K)}\right\| A \lambda_{i} J_{i}\right\rangle_{\mathrm{SD}},
\end{aligned}
$$

with the sum restricted to both $l+l^{\prime}+K$ and $l_{1}+l_{2}+K$ even. Eq. (16) is the desired relation between the physical translationally invariant density and the OBDME obtained in the Slater determinant basis. 


\section{Properties}

All the derivations presented in the preceding subsections were performed for a nucleon density. It should be noted that the Eq. (16) is trivially generalized to obtain the proton and the neutron densities separately. The relation (14) involves spatial transformations and remains valid even if we add spin or isospin operators. Similarly, the Eq. (16) is readily generalizable for a case of a nonlocal density that is needed as an input for some semi-microscopic optical potential folding approaches, see e.g. Ref. [16].

The physical density $(8,16)$ as well as the shell-model density $(5)$ are normalized as

$$
\int d \vec{x}\left\langle A \lambda J M\left|\rho_{o p}^{\mathrm{phys}}(\vec{x})\right| A \lambda J M\right\rangle=\int d \vec{x}{ }_{\mathrm{SD}}\left\langle A \lambda J M\left|\rho_{o p}^{\mathrm{SM}}(\vec{x})\right| A \lambda J M\right\rangle_{\mathrm{SD}}=A,
$$

where the superscripts "phys" and "SM" refer to Eqs. (8,16) and Eq. (5), respectively.

We note that the point-nucleon matter radius is obtained only by using the physical density

$$
r_{m}^{2}=\left\langle A \lambda J M\left|\frac{1}{A} \sum_{i=1}^{A}\left(\vec{r}_{i}-\vec{R}\right)^{2}\right| A \lambda J M\right\rangle=\frac{1}{A} \int d \vec{x} x^{2}\left\langle A \lambda J M\left|\rho_{o p}^{\text {phys }}(\vec{x})\right| A \lambda J M\right\rangle .
$$

The analogous integral for the shell-model density gives a value different from the point-nucleon matter radius as it contains a contribution from the spurious center of mass.

The present Eq. (16) can be compared to Eq. (7) of Ref. [9] and, similarly, Eq. (5) to Eq. (6) of Ref. [9]. In Ref. [9], the transformation that removes the CM components is made by inserting a complete set of non-spurious shell-model eigenstates between the $a^{\dagger}$ and $a$ operators which introduces the familiar $(A /(A-1))^{2 n+l / 2}$ factors that relate matrix elements in coordinates referred to the arbitrary origin and the $(\mathrm{A}-1)$ core. The present result is more general as a direct relation to the OBDME was found and, consequently, no sum of intermediate states is needed.

\section{APPLICATION TO ${ }^{6} \mathrm{HE}$}

We tested the physical translationally invariant density formulas (8) and (16) by performing identical calculations for ${ }^{3} \mathrm{H},{ }^{4} \mathrm{He}$ and ${ }^{5} \mathrm{He}$ in the Jacobi-coordinate $\mathrm{HO}$ basis and the Slater determinant HO basis, respectively. The Jacobi coordinate $\mathrm{HO}$ basis calculations were performed using the code MANYEFF [13] that constructs the $A$ nucleon antisymmetrized Jacobi coordinate HO basis, calculates the effective interaction from a nucleon-nucleon (NN) potential and, eventually, finds the $A$-nucleon eigenvalues and wave functions. These wave functions were then employed to calculate physical density according to Eq. (8). The same effective interaction, transformed to the singleparticle basis, was used in the Slater determinant HO basis calculation using the Many-Fermion Dynamics (MFD) [17] shell model code. A specialized code was then used to calculate the OBDME from the MFD eigenfunctions and eventually, the physical density was calculated by applying Eq. (16). We obtained identical results in the two independent calculations.

As an illustration of the significance of the spurious center-of-mass removal, we calculated the ${ }^{6} \mathrm{He}$ physical (16) and the shell-model (5) densities using wave functions obtained in Ref. [18]. In Fig. 1, the proton and the neutron monopole ground state densities are shown. A $10 \hbar \Omega$ basis space and the HO frequency of $\hbar \Omega=13 \mathrm{MeV}$ was used. The two-body effective interaction was derived from the CD-Bonn NN potential. The full lines correspond to the physical densities calculated according to Eq. (16) while the dashed lines correspond to the shell-model densities (5) that contain the spurious center-of-mass contribution. Obviously, the same OBDME were employed in both calculations. The normalization of the densities in Fig. 1 is $4 \pi \int d r r^{2} \rho_{K=0, p(n)}(r)=Z(N)$ with $p, n$ refers to the proton and neutron, respectively, and $\rho_{K=0}(r)=\frac{1}{4 \pi} \int d \hat{r}\left\langle A \lambda J M\left|\rho_{o p}(\vec{r})\right| A \lambda J M\right\rangle$. One can clearly see a substantial differences between the two sets of densities in particular at short distances. By performing the integral (18) for the physical density we indeed recover to point-proton and point-neutron rms radii $1.763 \mathrm{fm}$ and $2.361 \mathrm{fm}$, respectively [18]. Performing the same integral using the shell-model densities gives incorrect, larger radii $1.976 \mathrm{fm}$ and $2.524 \mathrm{fm}$, respectively. The difference between the squares of the two sets of radii is equal to the mean value of the CM $\vec{R}^{2}$, i.e. $\mathrm{SD}\left\langle A \lambda J M\left|\vec{R}^{2}\right| A \lambda J M\right\rangle_{\mathrm{SD}}=\frac{1}{A}\left\langle 000\left|\vec{\xi}_{0}^{2}\right| 000\right\rangle=0.798 \mathrm{fm}^{2}$

In Figs. 2 and 3 we present transition densities from the ${ }^{6} \mathrm{He}$ ground state to the first excited $2^{+}$state and the lowest $1^{-}$("soft-dipole mode") state, respectively. The $10 \hbar \Omega$ basis space was employed for the $0^{+} \rightarrow 2^{+}$case, while the $8 \hbar \Omega$ (for the ground state) and the $9 \hbar \Omega$ (for the $1^{-}$state) bases were utilized for the $0^{+} \rightarrow 1^{-}$transition. In both cases we can see substantial differences between the physical (16) and the shell model (5) densities. By integrating the proton densities, one obtains the reduced $E K$ matrix element:

$$
\int d \vec{x} x^{K} Y_{K, M_{f}-M_{i}}(\hat{x})\left\langle A \lambda_{f} J_{f} M_{f}\left|\rho_{o p, p}^{\mathrm{phys}}(\vec{x})\right| A \lambda_{i} J_{i} M_{i}\right\rangle=\frac{1}{\hat{J}_{f}}\left(J_{i} M_{i} K M_{f}-M_{i} \mid J_{f} M_{f}\right) M\left(E K ; J_{i} \rightarrow J_{f}\right)
$$


with $B\left(E K ; J_{i} \rightarrow J_{f}\right)=\frac{1}{\left(2 J_{i}+1\right)}\left|M\left(E K ; J_{i} \rightarrow J_{f}\right)\right|^{2}$. Here, the bare nucleon charges are assumed $\left(e_{p}=1, e_{n}=0\right)$. The result of the integral (19) is unchanged for the shell model densities, i.e. when the matrix element (16) is replaced by the matrix element (5) in Eq. (19). In the present particular cases we obtain $B\left(E 2 ; 0^{+} \rightarrow 2^{+}\right)=1.056 e^{2} \mathrm{fm}^{4}$ and $B\left(E 1 ; 0^{+} \rightarrow 1^{-}\right)=0.388 e^{2} \mathrm{fm}^{2}$ in agreement with the results found in ref. [18]. The normalization of the densities in Fig. 2 and 3 is $\int d r r^{2+K} \rho_{K, p}(r)=M(E K)$ for the proton case and similarly for the neutron case. Here, $\rho_{K}(r)=\hat{J}_{f} \sum_{M_{i} k}\left(J_{i} M_{i} K k \mid J_{f} M_{f}\right) \int d \hat{r} Y_{K}(\hat{r})\left\langle A \lambda_{f} J_{f} M_{f}\left|\rho_{o p}(\vec{r})\right| A \lambda_{i} J_{i} M_{i}\right\rangle$.

It has been argued that the spin-orbit component of the nucleon-nucleus optical potential is proportional to the derivative of the monopole density [19]. The following form of the potential that takes into account the isovector component is typically considered

$$
V_{\mathrm{so}}(r)=-(\pi / 3) V_{s} \frac{1}{r} \frac{d}{d r}\left[2 \rho_{K=0, p}(r)+\rho_{K=0, n}(r)\right] \vec{l} \cdot \vec{\sigma},
$$

for the proton-nucleus potential with the $p$ and $n$ indexes exchanged for the neutron-nucleus potential. In Eq. (20), $V_{s}$ (typically $V_{s}<0$ ) is a constant. We calculated the proton $-{ }^{6} \mathrm{He}$ spin-orbit optical potential according to Eq. (20) using the ${ }^{6} \mathrm{He}$ ground-state monopole densities shown in Fig. 1. The resulting shape of the spin-orbit potential, $\frac{1}{3} \frac{1}{r} \frac{d}{d r}\left[2 \rho_{K=0, p}(r)+\rho_{K=0, n}(r)\right]$, is presented in Fig. 4. In particular, we compare the result obtained using the physical density (16) with that obtained using the shell-model density (5) that includes the spurious center-of-mass contribution. Clearly, the differences of the densities as seen in Fig. 1 are even more magnified in the shape of spin-orbit potential. Such differences must have impact on observables like analyzing powers calculated using these spin-orbit potentials. In order to obtain meaningful results, one must employ the physical density to construct the spin-orbit component of the optical potential.

\section{CONCLUSIONS}

In this paper, the translationally invariant nuclear density was derived from the shell model one-body densities by removing their spurious $0 \hbar \Omega$ center-of-mass motion component. The main result of this paper, presented in Eq. (16) relates the translationally invariant density to the OBDME matrix elements calculated in the shell model codes by employing the second quantization techniques. This is important for the NCSM approach as calculations for $A>4$ are much more efficiently performed in the Cartesian-coordinate Slater determinant basis which has the down side of contaminating the wave functions by the spurious center-of-mass components. As the NCSM effective interaction is translationally invariant and for the basis space a complete $N_{\max } \hbar \Omega$ space is used, these components can always be exactly removed. In this paper, this was achieved for the density, a case less trivial compared to other operators. This paves the way to utilize the $a b$ initio no-core shell model nuclear structure in folding approaches to optical potentials.

We performed tests of the present formalism by performing independent Jacobi-coordinate HO basis and the Cartesian-coordinate HO Slater determinant basis for ${ }^{3} \mathrm{H},{ }^{4} \mathrm{He}$ and ${ }^{5} \mathrm{He}$. Identical results for the densities were obtained.

As an illustration, the ${ }^{6} \mathrm{He}$ diagonal and transitional densities were calculated from the NCSM wave functions obtained using the CD-Bonn nucleon-nucleon potential in the $10 \hbar \Omega$ basis space. These densities were compared to those obtained without the spurious center-of-mass component removal. Substantial differences were found for both the diagonal monopole and the transitional multipole densities. Only using the physical density one can recover the point-nucleon matter radius. On the other hand, the $E K$ reduced matrix elements can be obtained using both the physical and the uncorrected density. A particularly significant impact of the exact removal of the spurious centerof-mass motion was found for the spin-orbit part of the optical potential proportional to the derivative of the nuclear density.

The physical density can now be used in folding approaches to nucleon-nucleus optical potentials, such as those described, e.g. in Refs. [11] and [16], and subsequently applied in the coupled channel calculations. Work in this direction is under way.

\section{ACKNOWLEDGMENTS}

The author thanks D. J. Millener for useful comments. This work was performed under the auspices of the U. S. Department of Energy by the University of California, Lawrence Livermore National Laboratory under contract No. 
W-7405-Eng-48. Partial support from the LDRD contract No. 04-ERD-058 is acknowledged.

[1] B. S. Pudliner, V. R. Pandharipande, J. Carlson, S. C. Pieper and R. B. Wiringa, Phys. Rev. C 56, 1720 (1997); R. B. Wiringa, S. C. Pieper, J. Carlson, V. R. Pandharipande, Phys. Rev. C 62, 014001 (2000); S. C. Pieper and R. B. Wiringa, Ann. Rev. Nucl. Part. Sci. 51, 53 (2001); S. C. Pieper, K. Varga and R. B. Wiringa, Phys. Rev. C 66, 044310 (2002).

[2] P. Navrátil, J. P. Vary and B. R. Barrett, Phys. Rev. Lett. 84, 5728 (2000); Phys. Rev. C 62, 054311 (2000).

[3] E. Caurier, G. Martinez-Pinedo, F. Nowacki, A. Poves, J. Retamosa and A. P. Zuker, Phys. Rev. C 59, 2033 (1999); E. Caurier and F. Nowacki, Acta Physica Polonica 30, 705 (1999).

[4] L. J. Tassie and F. C. Barker, Phys. Rev. 111, 940 (1958).

[5] A. E. L. Dieperink and T. de Forest, Jr., Phys. Rev. C 10, 543 (1974).

[6] Taber de Forest, Jr., Phys. rev. C 22, 2222 (1980).

[7] R. J. Philpott, Nucl. Phys. A289, 109 (1977).

[8] Dean Halderson and R. J. Philpott, Nucl. Phys. A321, 295 (1979).

[9] D. J. Millener, J. W. Olness, E. K. Warburton and S. S. Hanna, Phys. Rev. C 28, 497 (1983).

[10] F. P. Brady, T. D. Ford, G. A. Needham, J. L. Romero, D. S. Sorenson, C. M. Castaneda, J. L. Drummond, E. L. Hjort, B. McEachern, N. S. P. King and D. J. Millener, Phys. Rev. C 43, 2284 (1991).

[11] J.-P. Jeukenne, A. Lejeune and C. Mahaux, Phys. Rev. C 16, 80 (1977).

[12] I. J. Thompson, Computer Physics Reports 7, 167 (1988).

[13] P. Navrátil, G. P. Kamuntavičius and B. R. Barrett, Phys. Rev. C 61, 044001 (2000).

[14] L. Trlifaj, Phys. Rev. C 5, 1534 (1972).

[15] G. R. Satchler, Direct Nuclear Reactions, Oxford University Press 1983.

[16] K. Amos, P. J. Dortmans, H. V. von Geramb, S. Karataglidis and J. Raynal, Advances in Nucl. Phys. 25, 275 (2000).

[17] J. P. Vary, "The Many-Fermion-Dynamics Shell-Model Code", Iowa State University (1992) (unpublished); J. P. Vary and D. C. Zheng, ibid., (1994).

[18] P. Navrátil, J. P. Vary, W. E. Ormand and B. R. Barrett, Phys. Rev. Lett. 87, 172502 (2001).

[19] R. R. Scheerbaum, Nucl. Phys. A257, 77 (1976); F. A. Brieva and J. R. Rook, Nucl. Phys. A297, 206 (1978); Ch. Lagrange, D. G. Madland and M. Girod, Phys. Rev. C 33, 1616 (1986); E. Bauge, J. P. Delaroche and M. Girod, Phys. Rev. C 58, 1118 (1998). 


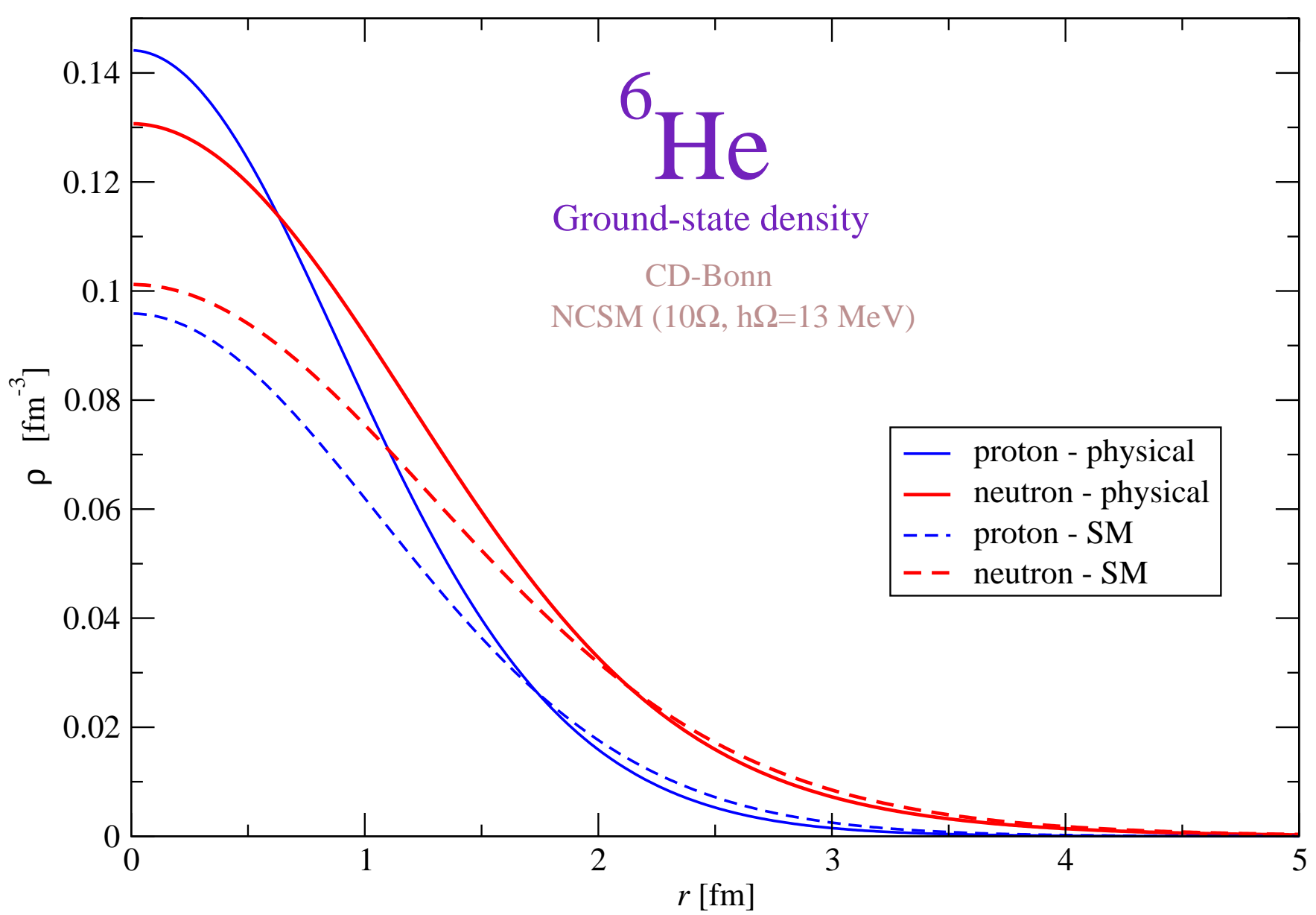

FIG. 1: (Color online) ${ }^{6} \mathrm{He}$ proton and neutron monopole ground state densities obtained in the $10 \hbar \Omega$ basis space and the HO frequency of $\hbar \Omega=13 \mathrm{MeV}$. The NCSM two-body effective interaction was derived from the CD-Bonn NN potential. The full lines correspond to the physical densities calculated according to Eq. (16) while the dashed lines correspond to the shell-model densities (5) that contain the spurious center-of-mass contribution. 


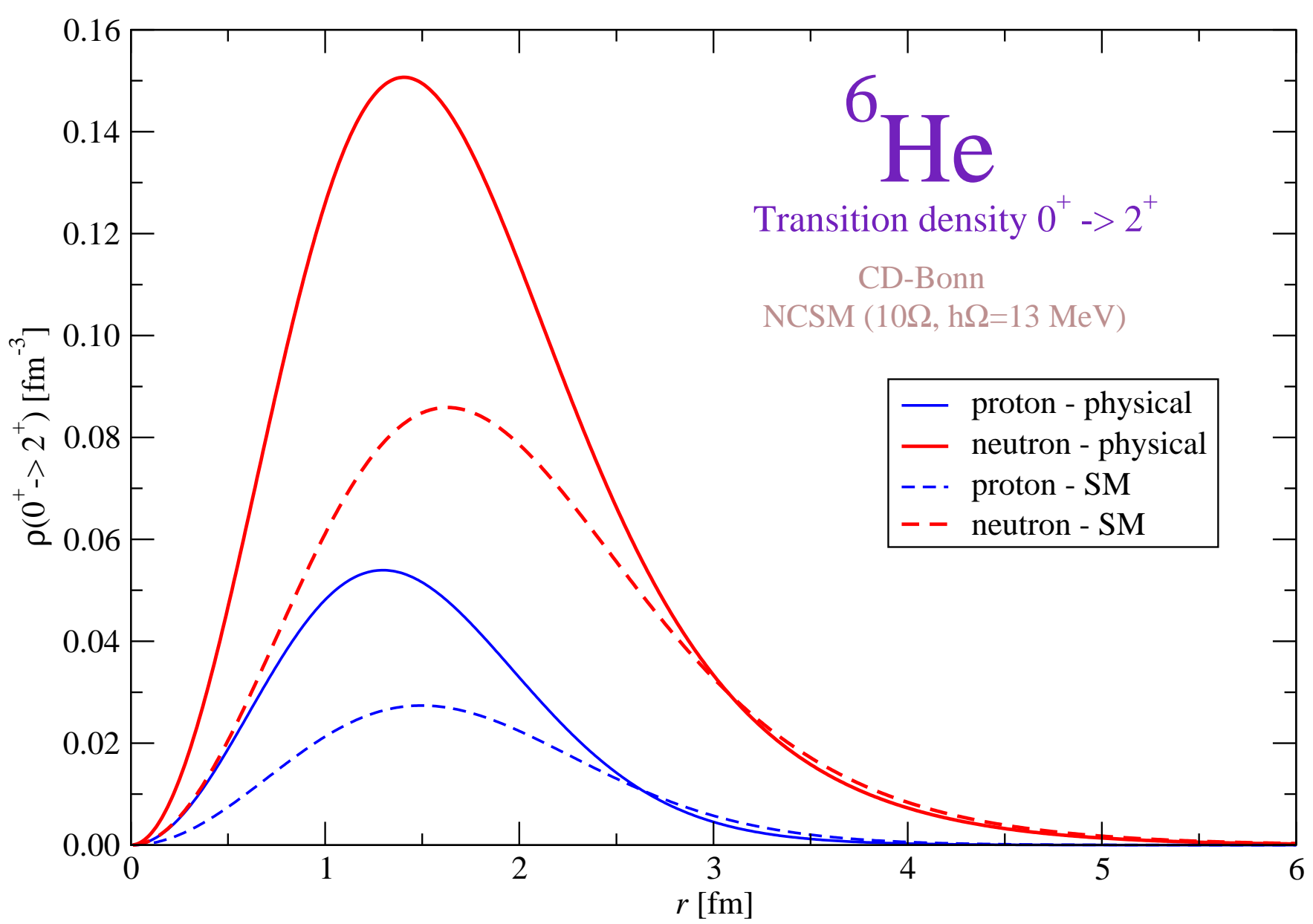

FIG. 2: (Color online) ${ }^{6} \mathrm{He}$ proton and neutron quadrupole transition densities from the ground state to the first excited $2^{+}$ state obtained in the $10 \hbar \Omega$ basis space and the HO frequency of $\hbar \Omega=13 \mathrm{MeV}$. The NCSM two-body effective interaction was derived from the CD-Bonn NN potential. The full lines correspond to the physical densities calculated according to Eq. (16) while the dashed lines correspond to the shell-model densities (5) that contain the spurious center-of-mass contribution. 


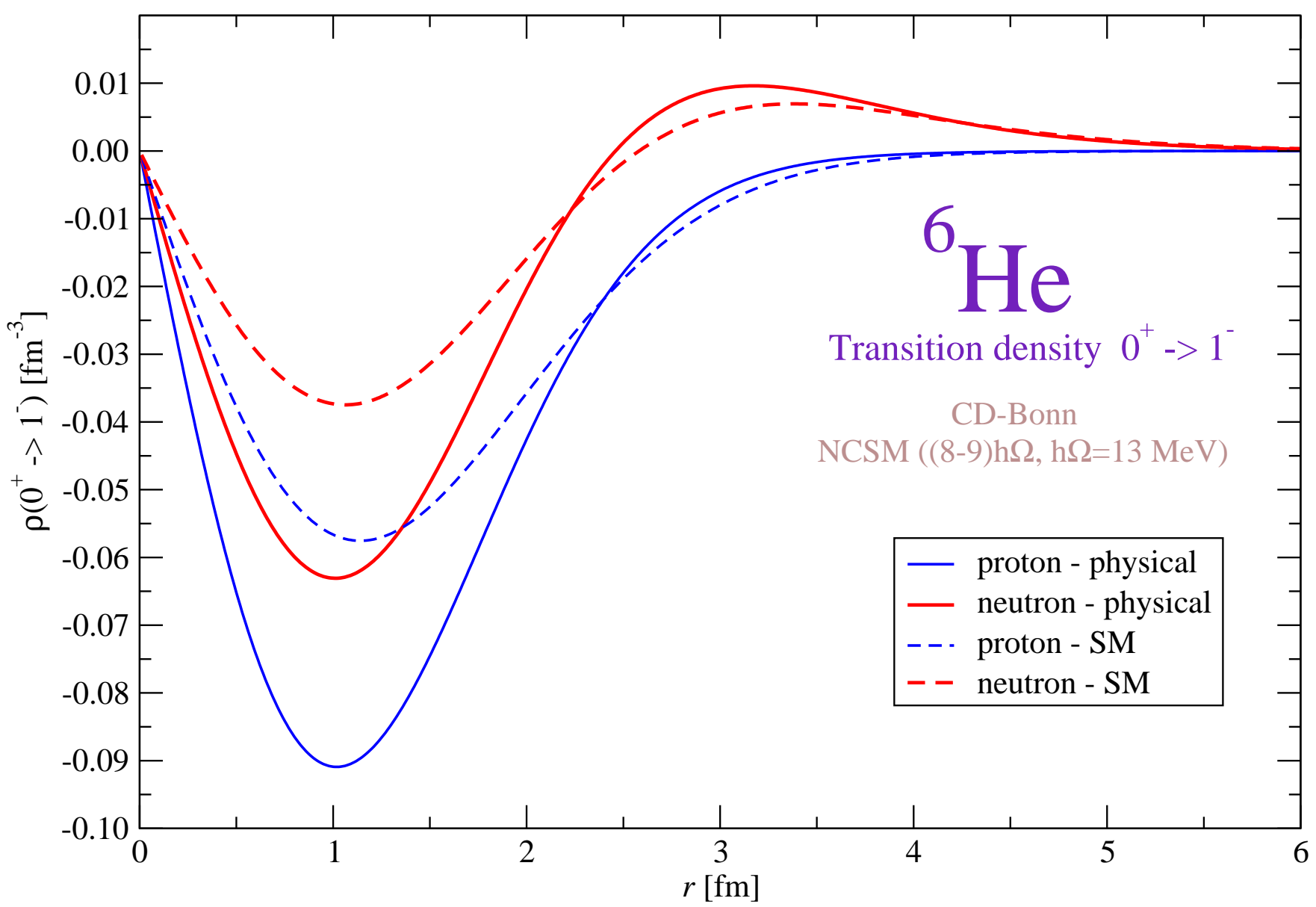

FIG. 3: (Color online) ${ }^{6} \mathrm{He}$ proton and neutron dipole transition densities from the ground state to the lowest $1^{-}$state obtained in the $(8-9) \hbar \Omega$ basis space and the HO frequency of $\hbar \Omega=13 \mathrm{MeV}$. The NCSM two-body effective interaction was derived from the CD-Bonn NN potential. The full lines correspond to the physical densities calculated according to Eq. (16) while the dashed lines correspond to the shell-model densities (5) that contain the spurious center-of-mass contribution. 


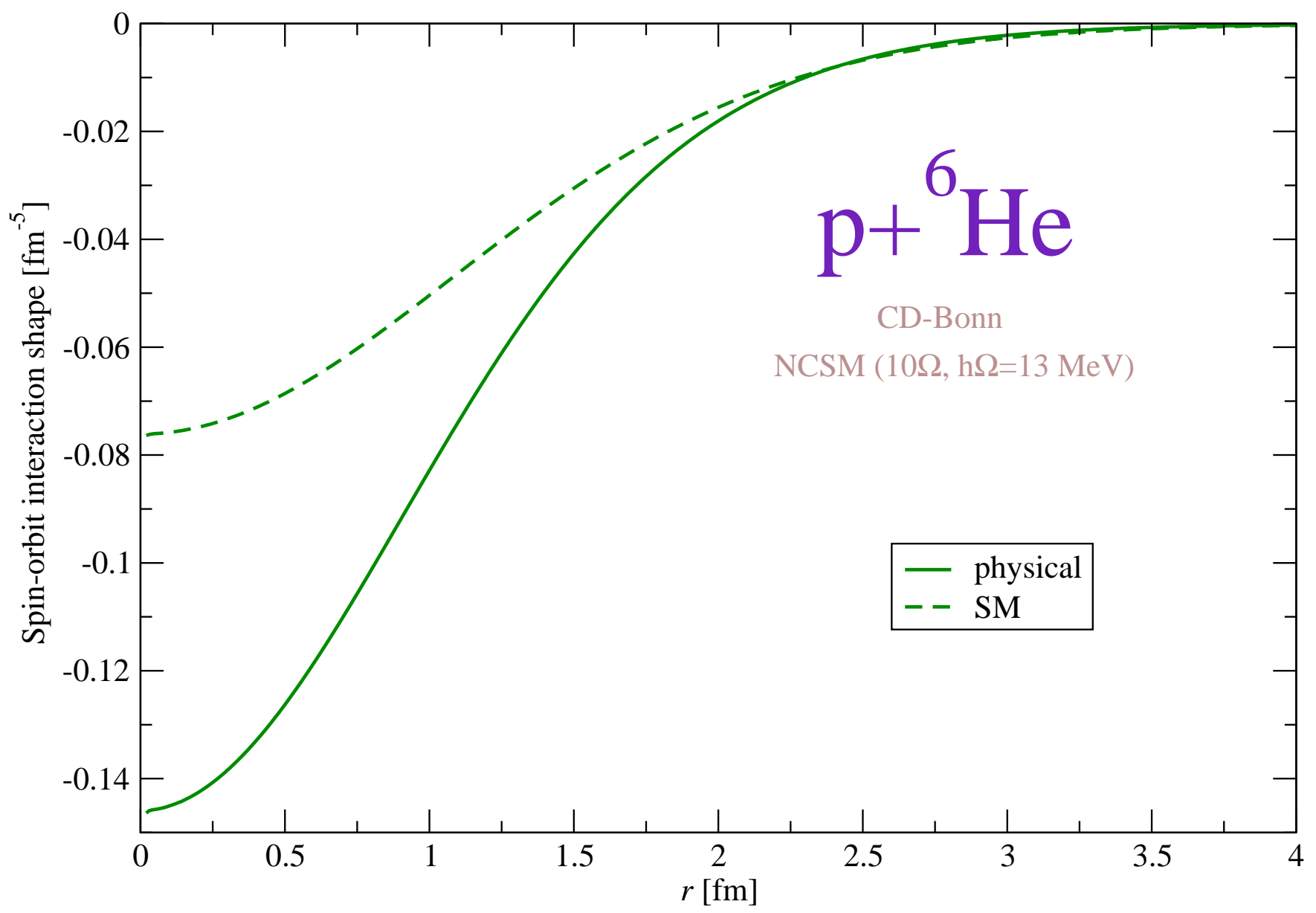

FIG. 4: (Color online) The shape of the proton ${ }^{6} \mathrm{He}$ spin-orbit optical potential, $\frac{1}{3} \frac{1}{r} \frac{d}{d r}\left[2 \rho_{K=0, p}(r)+\rho_{K=0, n}(r)\right]$, obtained using the ground state ${ }^{6} \mathrm{He}$ densities from the $10 \hbar \Omega, \hbar \Omega=13 \mathrm{MeV}$ NCSM calculation. The two-body effective interaction was derived from the CD-Bonn NN potential. The full lines correspond to the physical densities calculated according to Eq. (16) while the dashed lines correspond to the shell-model densities (5) that contain the spurious center-of-mass contribution. 


\section{Auspices Statement}

This work was performed under the auspices of the U.S. Department of Energy by University of California, Lawrence Livermore National Laboratory under Contract W-7405-Eng-48. 